\title{
ACCURACY OF POSITIONING AND ORIENTATION OF EFFECTOR OF PLANAR PARALLEL MANIPULATOR 3RRR
}

\author{
Monika PRUCNAL-WIESZTORT* \\ *Faculty of Mechanical Engineering, Wrocław University of Technology, ul. Łukasiewicza 5, 50-371 Wrocław, Poland \\ monika.prucnal@pwr.wroc.pl
}

received 25 November 2014, revised 9 October 2015, accepted 15 October 2015

\begin{abstract}
Parallel manipulator belongs to group of mechanisms with closed kinematic chains. This feature involves both advantages and disadvantages. The study examined the issue of accuracy of a planar system with three degrees of freedom, with revolute pairs, showing the effect of errors of the drives settings on effector positioning deviation. Enclosed is a numerical example for which analyzed the deviation in motion manipulator when going through the singular configuration. Based on the analysis was determined the area around the singular positions for which to obtain the orientation of the assumed accuracy is impossible.
\end{abstract}

Key Words: Accuracy, Planar Parallel Manipulator, Singularities

\section{INTRODUCTION}

Parallel manipulator belongs to group of mechanisms with closed kinematic chains. This feature involves both advantages and disadvantages. Desirable features include ability to transfer heavy loads, stiffness, accuracy of trajectory mapping (or positions), ability to gain high speed of operating element, which is result of good dynamic properties of the system due to possibility to placing of heavy active elements at the basis. But closure of the kinematic chain significantly reduce range of motion of the effector (Bikhari et al., 2014). Additionally, dividing the work zone, resulting from the range of possible positions of the intermediate elements and active elements on areas dependent on the mutual configuration of the system on subzones achievable for certain mutual configurations of the system elements, causes that moving between the subzones is possible after disassembly and assembly of the mechanism. Division into subzones is caused by occurrence of singular positions from direct kinematics inside of zone indicated by singular positions of inverse kinematics.

The particular configuration of the mechanism, called singular position, influencing on properties of the system - ceases to be effectively controllable (in this configuration the system loses its properties staring from motility, although they are characteristic within all other areas of the working zone). Singular positions appears in many analysis of parallel manipulator movements independently to adopted method of description (Bałchanowski, 2014).

\section{SCOPE OF WORK}

Scope of this study was to show the impact of the errors of the drive settings on deviation of effector's positioning and orientation. There was examined accuracy of parallel mechanism with three degrees of freedom with rotating kinematics pairs $-3 R R R$. Was done description of configuration of the mechanism and derived deviation equations. The whole was illustrated by an example. Purpose of attached numerical example was to show deviations of effector movement while passing trough singular positions.

\section{DESCRIPTION OF THE MANIPULATOR CONFIGUATION}

Studied parallel manipulator contains three kinematic chains connecting the effector 7 with the base (Fig. 1). Active elements 1 , 2, 3 are placed at the base. Intermediate elements 4, 5, 6 connect active elements with effector.

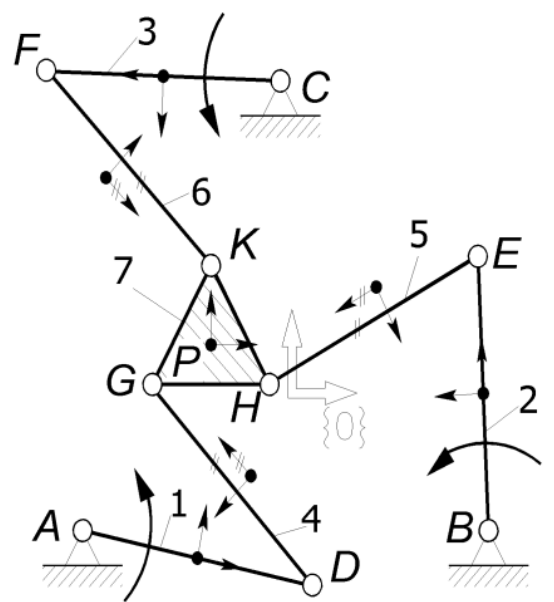

Fig. 1. The parameters of description of the position of elements in absolute coordinates

Description of the configuration of the mechanism was made using absolute coordinates. Every element was bound with local coordinate, which position and orientation described with vector $\boldsymbol{q}_{i}=\left[\begin{array}{lll}x_{i} & y_{i} & \Theta_{i}\end{array}\right]^{T}$ are the same as position of element $i$. Configuration of the mechanism is described then by the following system: 
$\Phi_{l}(\mathbf{q}, t)=\left[\begin{array}{l}\Phi^{W}(\mathbf{q}) \\ \Phi_{l}{ }^{C}(\mathbf{q}, t)\end{array}\right]=0$.

First group of equations $\Phi^{W}$ describes chains of kinematic pairs, second group is kinematic motion describing movement of active elements $\Phi_{l}{ }^{C}$, where $l=\{1,2\}$ depending on direct or inverse kinematic.

After differentiation of equation (1) velocity equation is given as

$\Phi_{l_{q}}(\mathbf{q}, t) \dot{\mathbf{q}}=-\Phi_{t} \equiv v$.

This record is compatible with the derivatives of the Lagrangian symbology, wherein the index t is the time derivative and $\Phi_{l_{q}}$ is jacobian $\Phi_{l_{q}}=\partial \Phi_{l} / \partial \mathbf{q}$.

After transformation equation (2) takes the form

$\dot{\mathbf{q}}=-\Phi_{l q}^{-1} \Phi_{t}$.

It should be noted that equation (3) has solution for $\operatorname{det}\left(\Phi_{l_{q}}\right) \neq 0$, otherwise there is singular position.

\section{ACCURACY ANALYSIS}

An important property of the system is accuracy of realization of the movement of the passive element (Briot and Bonev, 2008; Tannous et al., 2014; Yu et al., 2008; Zhang et al., 2014). For the known configuration it is possible to determine values which describe deviations resulting from drives setting errors.

Starting from equation (1) using the variance of function of pair chains $\Phi^{W}$, deviations are determined

$\Phi^{W}=0 \rightarrow \Phi_{\mathbf{q}}^{W} \delta \mathbf{q}=0$,

where: $\Phi_{\mathbf{q}}^{W}=\frac{\partial \Phi^{W}}{\partial \mathbf{q}}$.

Similar to vector of variables it is possible to distinguish vectors of conditional deviations and uninfluenced deviations as:

$\delta \mathbf{q}=\left[\begin{array}{ll}\delta \mathbf{q}_{Z}^{T} & \delta \mathbf{q}_{C}^{T}\end{array}\right]^{T}$.

Deviations vector $\delta \mathbf{q}_{C}$ is given value coming from type of used drivers and control system. After taking into account (5) equation (4) transforms to

$\Phi_{q Z}^{W} \delta \mathbf{q}_{Z}+\Phi_{q C}^{W} \delta \mathbf{q}_{C}=0$

and allows to find conditional deviations from equation:

$\delta \mathbf{q}_{Z}=-\left[\Phi_{q Z}^{W}\right]^{-1} \Phi_{q C}^{W} \delta \mathbf{q}_{C}$

the equation has unique solution for $\operatorname{det}\left(\Phi_{q Z}^{W}\right) \neq 0$.

Equation (7) allows to determine deviations of point $P$ in relation to nominal positions under condition of knowledge of value and sign of drives setting error $\delta \mathbf{q}_{C}$. Because at stage theoretical considerations information about used drive and control system (errors of setting of active elements) is not available, then the maximum deviation values are determined resulting from errors of setting of $n$ drives basing on relation (8)

$\delta x_{P \max }=\sum_{i=1}^{3}\left|w_{x i} \delta q_{C i}\right|$

$\delta y_{P \max }=\sum_{i=1}^{3}\left|w_{y i} \delta q_{C i}\right|$.
In equation (8) values of $\delta \mathbf{q}_{i}$ are known, and values of $w_{x i}$, $w_{y i}, w_{\Theta_{3 i}}$ are influence factors defined according to equations:

$w_{x i}=\frac{\delta x_{P}}{\delta q_{C i}}$

$w_{y i}=\frac{\delta y_{P}}{\delta q_{C i}}$.

$w_{\Theta_{3 i}}=\frac{\delta \Theta_{3}}{\delta q_{C i}}$

Values of influence factors from individual drives are determined basing on known components of vector $\delta \mathbf{q}_{C}$, while calculating influence of setting error of drive $k \delta \mathbf{q}_{c_{k}}$ ), remaining two has value of zero: $\delta \mathbf{q}_{c_{m}}=\delta \mathbf{q}_{c_{j}}=0$ for $m, j \neq k$.

Value of influence factor depends only on configuration of the system and allows the accuracy analyze independent from values of errors of setting of individual drives.

\section{CALCULATION EXAMPLE}

Model described above was implemented into mechanism described on Fig. 2, which dimensions are presented in Tab. 1.

Tab. 1. Dimensions of the analyzed manipulator

\begin{tabular}{|l|c|c|}
\hline Element & symbol & $\mathrm{mm}$ \\
\hline Base & $A B=B C=C A$ & 173 \\
\hline Crank & $l_{1}=l_{2}=l_{3}$ & 200 \\
\hline Connector & $l_{4}=l_{5}=l_{6}$ & 200 \\
\hline Effector & $G H=H K=K G$ & 100 \\
\hline
\end{tabular}

In order to use method described above, condition of solution of equation (7) must be checked. This equation has solution for $\operatorname{det}\left(\Phi_{q Z}^{W}\right) \neq 0$ (Choi et al., 2013; Firmani and Podhorodeski, 2009; Huang and Thebert, 2010). Following is determinant of the matrix $\Phi_{q Z}^{W}$ (Gronowicz and Prucnal-Wiesztort, 2006):

$\operatorname{det}\left(\Phi_{q Z}^{W}\right)=\left({ }^{4} x_{G}-{ }^{4} x_{D}\right)\left({ }^{5} x_{H}-{ }^{5} x_{E}\right)\left({ }^{6} x_{K}-{ }^{6} x_{F}\right)$

$\left.\left\{\sin \left(\Theta_{6}-\Theta_{5}\right)\left({ }^{7} x_{G} \sin \left(\Theta_{4}-\Theta_{7}\right)\right)-{ }^{7} y_{G} \cos \left(\Theta_{4}-\Theta_{7}\right)\right)\right)$

$\left.+\sin \left(\Theta_{4}-\Theta_{6}\right)\right)\left({ }^{7} x_{H} \sin \left(\Theta_{5}-\Theta_{7}\right)-{ }^{7} y_{H} \cos \left(\Theta_{5}-\Theta_{7}\right)\right)$

$\left.\left.+\sin \left(\Theta_{5}-\Theta_{4}\right)\right)\left({ }^{7} x_{K} \sin \left(\Theta_{6}-\Theta_{7}\right)-{ }^{7} y_{K} \cos \left(\Theta_{6}-\Theta_{7}\right)\right)\right\}$

which corresponds to the value of determinant of Jacobian matrix for direct kinematic.

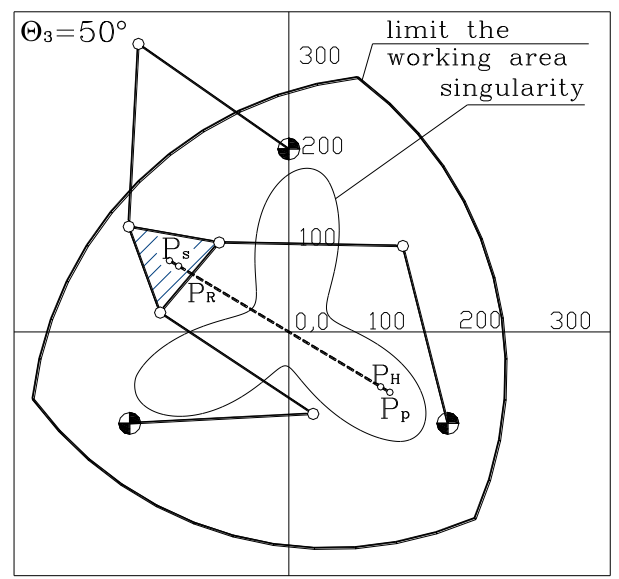

Fig. 2. Realized trajectory 
Singular positions of direct kinematic are possible to determine for planar kinematic systems with three degree of freedom using their graphic interpretation (Fig. 3).
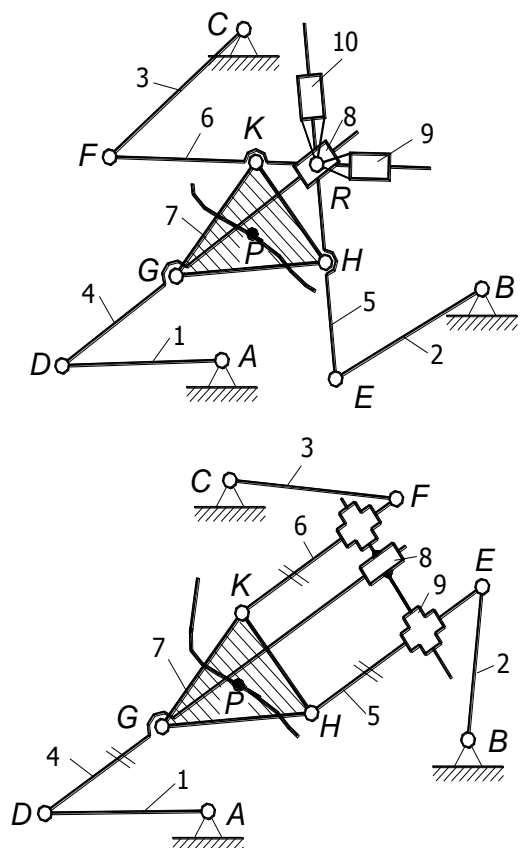

Fig. 3. Supporting mechanisms

After implementation of additional elements, mobility of supporting mechanisms for: $n=11, p 1=14, p 2=0$ is equal to:

$W=3(n-1)-2 p_{1}-p_{2}=3 \cdot 10-14 \cdot 2=2$.

For known orientation of effector degree of freedom is 1 , which indicates continuity of singular configuration for given orientation. Working area for chosen orientation of effector with singular positions is presented on Fig. 4.

In this situation answer for question about value of deviations of positions and orientation of effector caused by errors of setting of the drives require specific analyze. Because usage of equation (7) throughout the range of motion expected it was decided, that analyze basing on solving of direct kinematic for nominal values of setting of the drives will be performed, and then the solution of a direct kinematic for all conceivable variations of displacement settings of the three drives. This is the basis for the adoption of additional procedures for analyzing all the deviations and selecting maximum disadvantageous of their systems, which in turn allows to specify the range of possible trajectory deviations. For this purpose algorithm was built (Fig. 5). Analyze of the accuracy of realization of the trajectory was determined as the difference between the nominal values of the position and orientation of the effector and the designated coordinates of the point $\mathrm{P}$ and the orientation $\Theta_{3}$ of specific effector assumed with misalignment of the drives. Near the singular positions algorithm for the positions go on polynomial record, where the variable was the angle of orientation of the platform $\Theta_{3}$. The displacement values of the drives set were assumed on the level of $\Delta u_{i}= \pm 0.001 \mathrm{rad}$.

In Tab. 2 were shown parameters of realized trajectory, where $P_{S}$ is starting point, $P_{R}$ is the point from which traffic moves at a constant speed $v_{P}, P_{H}$ - the point at which braking occurs,

In the Tab. 2 shows the parameters of the realized trajectory, the $P_{S}$ is the starting point, $P_{R}$ point from which traffic is moving at a constant velocity $v_{P}, P_{H}$ - the point at which braking occurs, $P_{P}$ - staging point lasting for the duration of $T_{P}$, followed by return to the $P_{S}$.

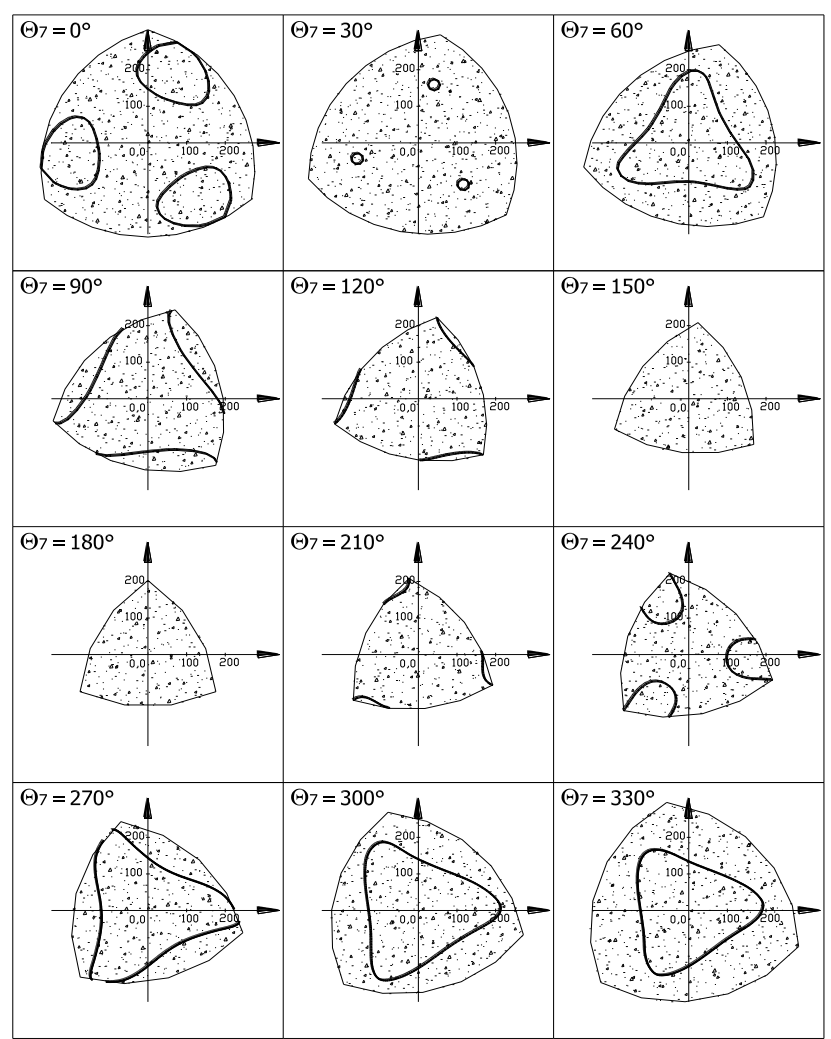

Fig. 4. Singular position against the work area

Tab. 2. Parameters of trajectory

\begin{tabular}{|c|c|c|c|c|c|}
\hline $\begin{array}{c}P_{S}(x, y) \\
\mathrm{mm}\end{array}$ & $\begin{array}{c}P_{R}(x, y) \\
\mathrm{mm}\end{array}$ & $\begin{array}{c}P_{H}(x, y) \\
\mathrm{mm}\end{array}$ & $\begin{array}{c}P_{P}(x, y) \\
\mathrm{mm}\end{array}$ & $\begin{array}{c}T_{P} \\
\mathrm{~s}\end{array}$ & $\begin{array}{c}v_{P} \\
\mathrm{~m} / \mathrm{s}\end{array}$ \\
\hline$-130,78$ & $-120,72$ & $100,-60$ & $110,-66$ & 3 & 0.06 \\
\hline
\end{tabular}

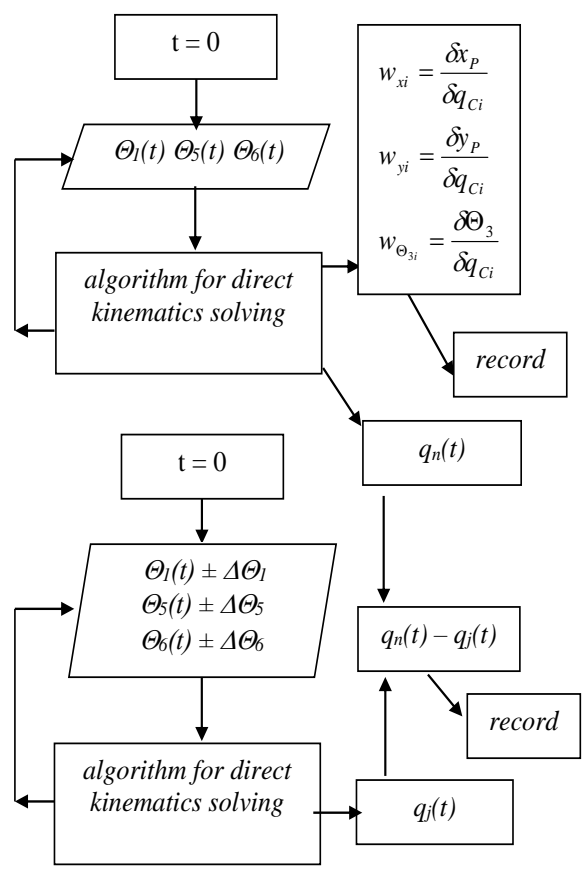

Fig. 5. A block diagram of algorithm accuracy 
The results show that the trajectory in the working area with singular positions deviation of positioning and orientation of the effector dramatically increases. Fig. 6 shows the resulting deviation of the effector orientation.

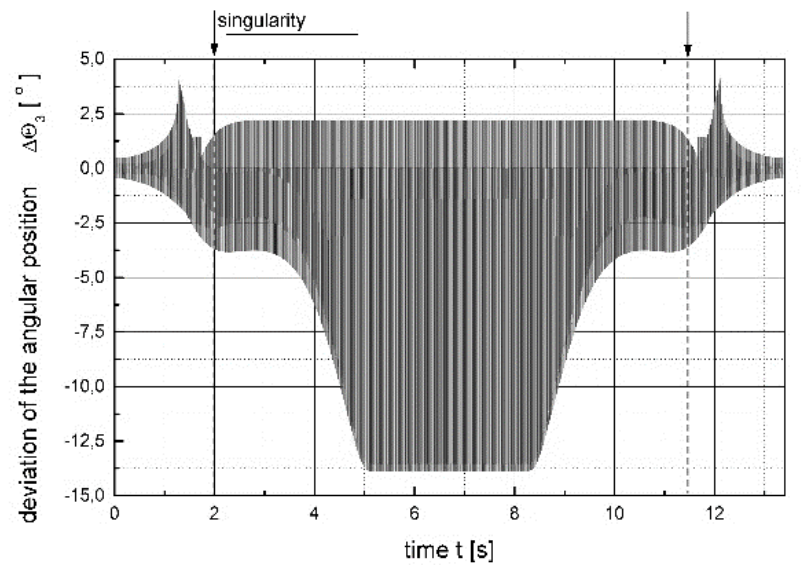

Fig. 6. Deviation of orientation $\Delta \Theta_{3}$

Permissible error of effector orientation was adopted at 30 . Such an approach entails a condition limiting the permissible zone.

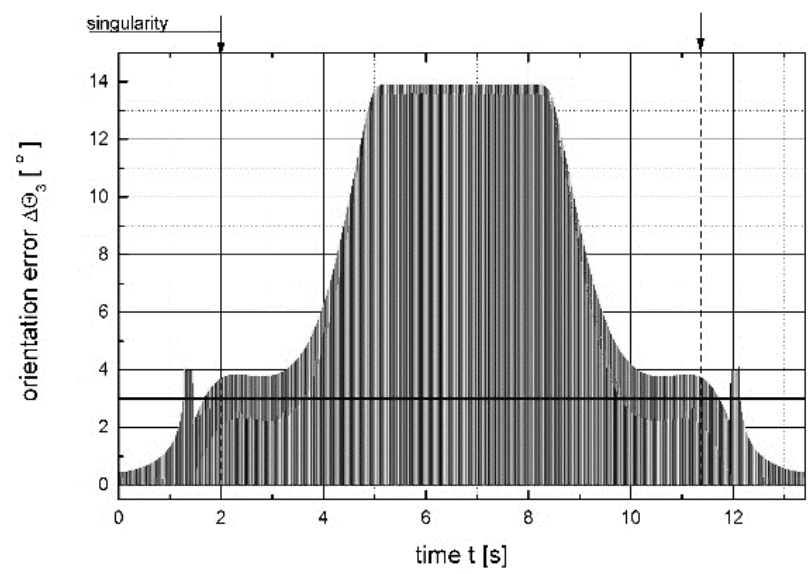

Fig. 7. Deviation of orientation during effector movement $\Delta \Theta_{3}$

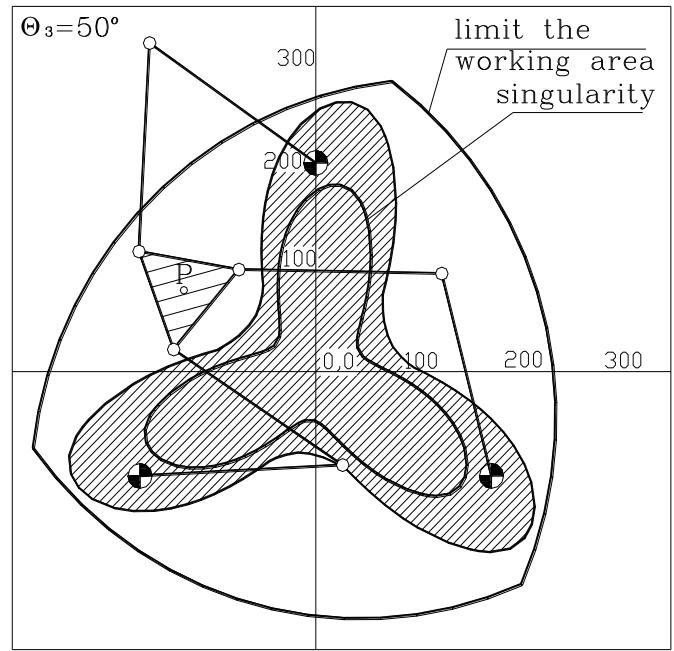

Fig. 8. Permissible working area for orientation of effector $\Delta \Theta_{3}=50^{\circ}$ (criterion of accuracy)
Basing on the analysis was defined area around the singular positions, for which achievement of orientation at predetermined accuracy is not possible (hatched in Fig. 8).

Working area for the orientation of the effector $\Delta \Theta_{3}=50^{\circ}$ has been significantly reduced. Practically the entire central portion is not available.

\section{CONCLUSION}

The study examined the issue of accuracy of the parallel mechanism with three degrees of freedom $-3 R R R$. After derivation of equation of the deviation a numerical example was presented, for which is given manipulator working zone with different orientations including singular positions of the direct kinematics. Purpose of attached numerical example was to show deviations of effector movement while passing trough singular positions.

Based on the analysis was determined the area around the singular positions for which to obtain the orientation of the assumed accuracy is impossible (hatched in Fig. 8). Work area for effector orientation $\Delta \Theta_{3}=50^{\circ}$ has been significantly reduced. Practically the entire central portion is not available. This points to the need for a significant limitations and such a small work area of analyzed manipulator, which may preclude it from practical applications.

\section{REFERENCES}

1. Bałchanowski J. (2014), Topology and analysis of the singularities of a parallel mechanism with three degrees of freedom, Archives of Civil \& Mechanical Engineering, 14(1), 80-87.

2. Banke B., Dhiraj K., Chandan J., Vijay S. (2014), Rathore and Anjan Kumar Dash A geometric approach for the workspace analysis of two symmetric planar parallel manipulators, Robotica, FirstView, 1- 26.

3. Briot S., Bonev I. A. (2008), Accuracy analysis of 3-DOF planar parallel robots, Mechanism and Machine Theory, 43(4), 445-458.

4. Choi J. H., Seo T., Lee J. W. (2013), Singularity Analysis of a Planar Parallel Mechanism with Revolute Joints based on a Geometric Approach, International Journal of Precision Engineering and Manufacturing, 14(8), 1369-1375.

5. Firmani F., Podhorodeski R. P. (2009), Singularity analysis of planar parallel manipulators based on forward kinematic solutions, Mechanism \& Machine Theory, 44 (7), 1386-1399.

6. Gronowicz A., Prucnal-Wiesztort M. (2006), Singular configurations of planar paralel manipulators, Archves of Civil and Mechanical Engineering, 6(2), 21-30.

7. Huang M. Z., Thebert J.-L. (2010), A study of workspace and singularity characteristics for design of 3-DOF planar parallel robots, International Journal of Advanced Manufacturing Technology, 51(5-8), 789-797.

8. Tannous M., Caro S., Goldsztejn A. (2014), Sensitivity analysis of parallel manipulators using an interval linearization method, Mechanism \& Machine Theory, 71, 93-114.

9. Yu A., Zsombor-Murray P., Bonev I. A. (2008), Geometric approach to the accuracy analysis of a class of 3-DOF planar parallel robots, Mechanism and Machine Theory, 43(3), 364-375.

10. Zhang Q. H, Fan X. R., Zhang X. M. (2014), Dynamic Analysis of Planar 3-(R)under-barRR Flexible Parallel Robots with Dynamic Stiffening, Shock and Vibration, Article ID 370145, 1-13. 